\title{
Detection of extended spectrum beta lactamase from multidrug resistance Escherichia coli from various clinical sample in District Peshawar, Pakistan
}

\author{
Muhammad Afzaal ${ }^{1}$, Mohammad Haroon ${ }^{2}$, Muhammad Farooq Afridi ${ }^{1}$, \\ Abdul Haseeb ${ }^{1}$, Faaiz ul Hassan ${ }^{1}$, Muhammad Zahid ${ }^{3}$, Mudassir Shah ${ }^{1 *}$, \\ Muhammad Sajid ${ }^{3}$ and Riaz Ahmad ${ }^{3}$ \\ 1. Department of Zoology, Government Superior Science College Peshawar, KPK-Pakistan \\ 2. Department of Medicine, Khyber Teaching Hospital Peshawar, KPK-Pakistan \\ 3. Department of Zoology, Islamia College University Peshawar, KPK-Pakistan \\ *Corresponding author's email: mshahsafi75@gmail.com
}

Citation

Muhammad Afzaal, Mohammad Haroon, Muhammad Farooq Afridi, Abdul Haseeb, Faaiz ul Hassan, Muhammad Zahid, Mudassir Shah, Muhammad Sajid and Riaz Ahmad. Detection of extended spectrum beta lactamase from multidrug resistance Escherichia coli from various clinical sample in District Peshawar, Pakistan. Pure and Applied Biology. Vol. 9, Issue 4, pp2383-2390. http://dx.doi.org/10.19045/bspab.2020.90252

\begin{tabular}{llll}
\hline \hline Received: 09/03/2020 & Revised: 16/06/2020 & Accepted: 17/07/2020 & Online First: 20/07/2020 \\
\hline
\end{tabular}

\section{Abstract}

Gram negative bacteria's such as Escherichia coli is one of the most common community-acquired as well as the nosocomial pathogen responsible for a large number of infections. Extendedspectrum beta-lactamase producing strains of Escherichia coli has become a great therapeutic challenge to the clinicians in managing such infections. This study was carried out in order to find out multidrug-resistant Escherichia coli from various clinical samples. A total of 70 E. coli isolates were obtained from various clinical samples. These already identified samples were obtained from Lady Reading Hospital. Antibiotic susceptibility test was done by disc diffusion method. The present study shows that $(55.71 \%)$ samples were ESBL positive. The percentage of isolates sensitive to current antibiotics were highest for Polymyxin b (98.57\%) and highest for Ceftriaxone $(12.85 \%)$. While the percentage of isolates resistant to current antibiotics were highest for that Cotrimoxazole (84.28\%), and lowest for Polymyxin b (1.42\%). ESBL producing strains of $E$. coli causes therapeutic failure and also contribute to multidrug resistance. Therefore monitoring of antimicrobial resistance in developing countries is necessary to optimize empiric treatment and the prudent use of antimicrobials.

Keywords: Escherichia coli; Extended-spectrum $\beta$-lactamase; Multidrug resistance

\section{Introduction}

Escherichia coli is one of the most common community-acquired pathogens responsible for a large number of nosocomial infections
[1]. E. coli is a gram-negative, anaerobic, rod-shaped, coliform bacterium of the genus Escherichia, commonly occur as normal flora of small intestine of warm-blooded 
organisms [2]. The majority of $E$. coli strains are harmless. These harmless strains are part of the normal flora of the gut [3-5], which are benefited by producing vitamin $\mathrm{K}$ in their hosts [6] and prevent the colonization of the intestine with other pathogenic bacteria [7].

The E. coli related disease for the first time is diagnosed in 1982 from undercooked cow meat and unpasteurized milk [8]. The transmission of E.coli occurs through contaminated water (water which comes in contact with the feces of animals), while using it for drinking, for washing vegetables and fruits, and swimming in contaminated pools and lakes. It is important to note that the age and immune system of a person affected by a disease play a major role in its progress. Recent studies show's that infants aged younger than 5 years are more susceptible to this bacterium. Eating undercooked meat and burgers, eating on surfaces at service restaurants, using the immune-suppressing medication can attract $E$. coli related disease [9]. Most of the E. coli strains do not cause any disease [10], but virulent strains are responsible for gastroenteritis, urinary tract infections, and neonatal meningitis. It can also cause severe abdominal cramps, diarrhea that normally turns bloody within 24 hours, and sometimes fever. In rarer cases, virulent strains are also responsible for bowel necrosis (tissue death) and perforation without progressing to the hemolytic-uremic syndrome, peritonitis, mastitis, septicemia, and Gram-negative pneumonia [11]. The Urinary tract infection is mostly caused by E. coli. [12].

The frequent use of antibiotics in rural farms increases the antimicrobial resistance in developing countries, which promotes the multiple drug resistance (MDR) in E. coli in both human and veterinary medicines [13, 14]. In clinical settings, mostly E.coli strains are treated $\beta$-lactam antibiotics, the extensive use of which has led to the emergence of ESBL's producing strains that can hydrolyze $\beta$-lactam antibiotics [15]. The ESBLs are chromosomal or plasmid-mediated $\beta$ lactamases (Enzymes that cleave the $\beta$ lactam ring), have mutated from pre-existing broad- spectrum $\beta$-lactamases (TEM-1, TEM-2, SHV-1), as a consequence of frequent use of 3 rd generation antibiotics like Cephalosporins and Aztreonam. ESBLproducing genes are normally found on plasmids and its size is $80 \mathrm{~kb}$ or larger which carry resistance determinants for aminoglycosides, fluoroquinolones, tetracyclines, Chloramphenicol and Cotrimoxazole, making the micro-organisms resist a wide variety of antibiotics [16]. Several different methods have been used for the detection of ESBL producing strains, but the most widely used technique is Doubledisk synergy or disk approximation [17].

Although bacterial resistance to broadspectrum $\beta$ - lactam antibiotics are major concerns and the primary focus for clinicians and researcher, until recently not many studies have conducted in Peshawar, Pakistan to detect ESBL's producing $E$ coli strains. The present study was conducted with an aim to determine the ESBL enzyme in multidrugresistant $E$. coli isolated from various clinical samples.

\section{Materials and methods Study design}

Prospective study was designed to determine the ESBL enzyme in multidrug-resistant $E$. coli isolated from various clinical samples.

\section{Sampling}

The study was centered on 70 samples of $E$. coli, which were collected from Lady Reading Hospital (LRH) in Peshawar, during the period of (February 2018 to August 2018). The Samples were obtained using sterile techniques to circumvent contamination

\section{Antibiotic sensitivity testing}

Antibiotic susceptibility test was done by applying the Kirby-Bauer disk diffusion method, according to the Clinical and 
Laboratory Standard Institute (CLSI). Commercially available antibiotic discs were used. For example Amikacin (AK), Cefepime (FEP), Ciprofloxacin (CIP), Ceftriaxone (CRO), Amoxicillin (AMC), Tazobactam (TZP), Imipenem (IPM), Co-trimoxazole (SXT) and Polymyxin b (PB).

\section{Phenotypic detection of ESBL}

ESBL production was confirmed phenotypically by Double-disc synergy test (DDST) according to the Clinical and Laboratory Standards Institute (CLSI) criteria for ESBL screening. According to the CLSI protocol, DDST was done by using amoxicillin $(30 \mu \mathrm{g}) /$ clavulanic acid $(10 \mu \mathrm{g})$ and cefepime $(30 \mu \mathrm{g})$. The discs were placed $25 \mathrm{~mm}$ apart from each other on MullerHinton agar (MHA) plate inoculated with 0.5 McFarland suspension of the tested isolates. The plate was incubated overnight at $37^{\circ} \mathrm{C}$. AMC may or may not be sensitive, and FEP will be resistant but will be sensitive upto some extent towards AMC disc, which is due synergistic effect of clavulanic acid. The window formation indicated the presence of ESBL

\section{Statistical analysis}

All the data were presented in graphs and figures and was expressed in percentages.

\section{Results}

A total of 70 identified samples of E. coli were collected from the patients with the age 1day-90years, out of which $32(45.71 \%)$ males and $38(54.28 \%)$ females. Different specimens e.g urine, pus, wound swab were received from the indoor or admitted patients in Lady reading hospital Peshawar KPK (Fig. 1) depicts the sample wise distribution of clinical isolates of $E$. coli.

Antibiotic susceptibility testing in our isolates, we have found increased percentage $(98.57 \%)$ of isolates showed sensitivity to polymyxin $b$ followed by imipenem, which showed sensitivity of (90\%). $84.28 \%$ of $E$ .coli isolates showed resistance to co.trimoxazole. However, we have observed an elevated level of resistance to other routinely used antibiotics. The Growth of Resistant E. coli strain has been shown (Fig. 2 ), while cumulative susceptibility pattern of E.coli isolates were shown (Table 1).

The resistant antibiotic strains were tested for their ability to produce ESBL, the percentage of which has been shown (Fig. 3), while ESBL positive strain is shown (Fig. 4).

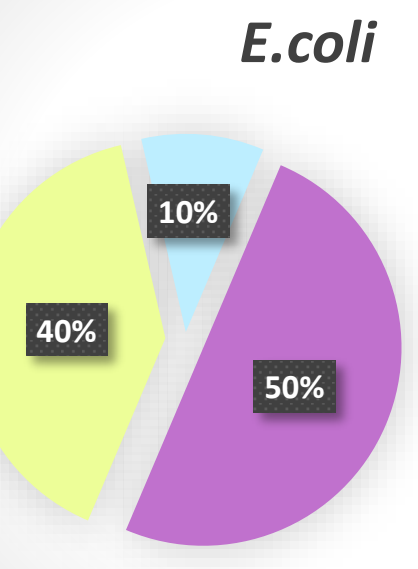

Wound Swab Urine Pus

Figure 1. Shows the Percentage of $E$. coli in various clinical samples 


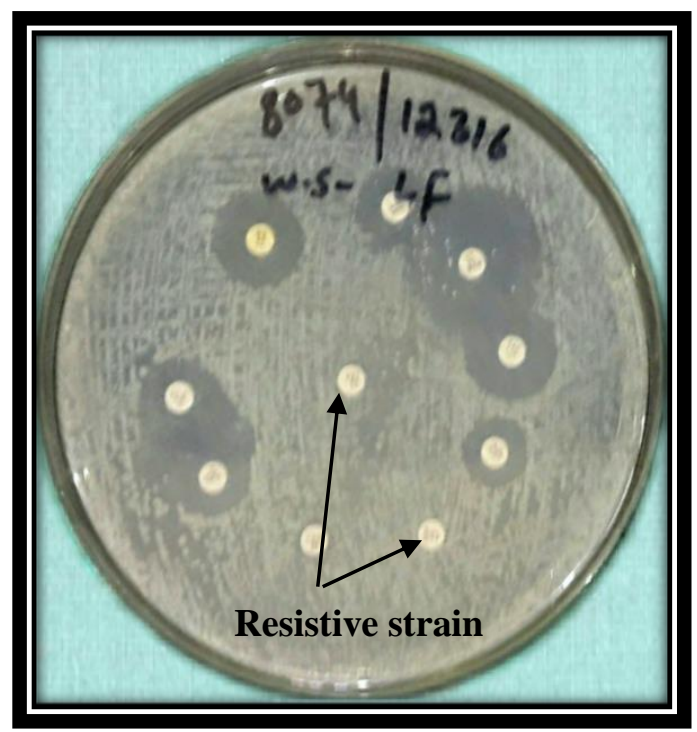

Figure 2. Growth of Resistant $E$. coli strain on MHA media

Table 1. Cumulative susceptibility patterns of $E$. coli to current antibiotics

\begin{tabular}{|c|c|c|c|c|c|c|c|}
\hline \multirow{2}{*}{ Antibiotic disk } & \multirow{2}{*}{ Total no } & \multicolumn{2}{|c|}{ Sensitive } & \multicolumn{2}{c|}{ Resistance } & \multicolumn{2}{c|}{ Intermediate } \\
& & No & \% & \multicolumn{2}{c|}{ No } & No & \% \\
\hline Amoxicillin & 70 & 43 & $61.42 \%$ & 24 & $34.28 \%$ & 3 & $4.28 \%$ \\
\hline Cefepime & 70 & 43 & $61.42 \%$ & 25 & $35.71 \%$ & 2 & $2.85 \%$ \\
\hline Ceftriaxone & 70 & 9 & $12.85 \%$ & 49 & $70 \%$ & 12 & $17.14 \%$ \\
\hline Ciprofloxacin & 70 & 11 & $15.71 \%$ & 53 & $75.71 \%$ & 6 & $8.57 \%$ \\
\hline Imipenem & 70 & 63 & $90 \%$ & 4 & $5.71 \%$ & 3 & $4.28 \%$ \\
\hline Amikacin & 70 & 52 & $74.28 \%$ & 5 & $7.14 \%$ & 13 & $18.57 \%$ \\
\hline Tazobactam & 70 & 56 & $80 \%$ & 5 & $7.14 \%$ & 9 & $12.85 \%$ \\
\hline Co-trimoxazole & 70 & 10 & $14.28 \%$ & 59 & $84.28 \%$ & 1 & $1.42 \%$ \\
\hline Polymyxin b & 70 & 69 & $98.57 \%$ & 1 & $1.42 \%$ & 0 & $0 \%$ \\
\hline
\end{tabular}

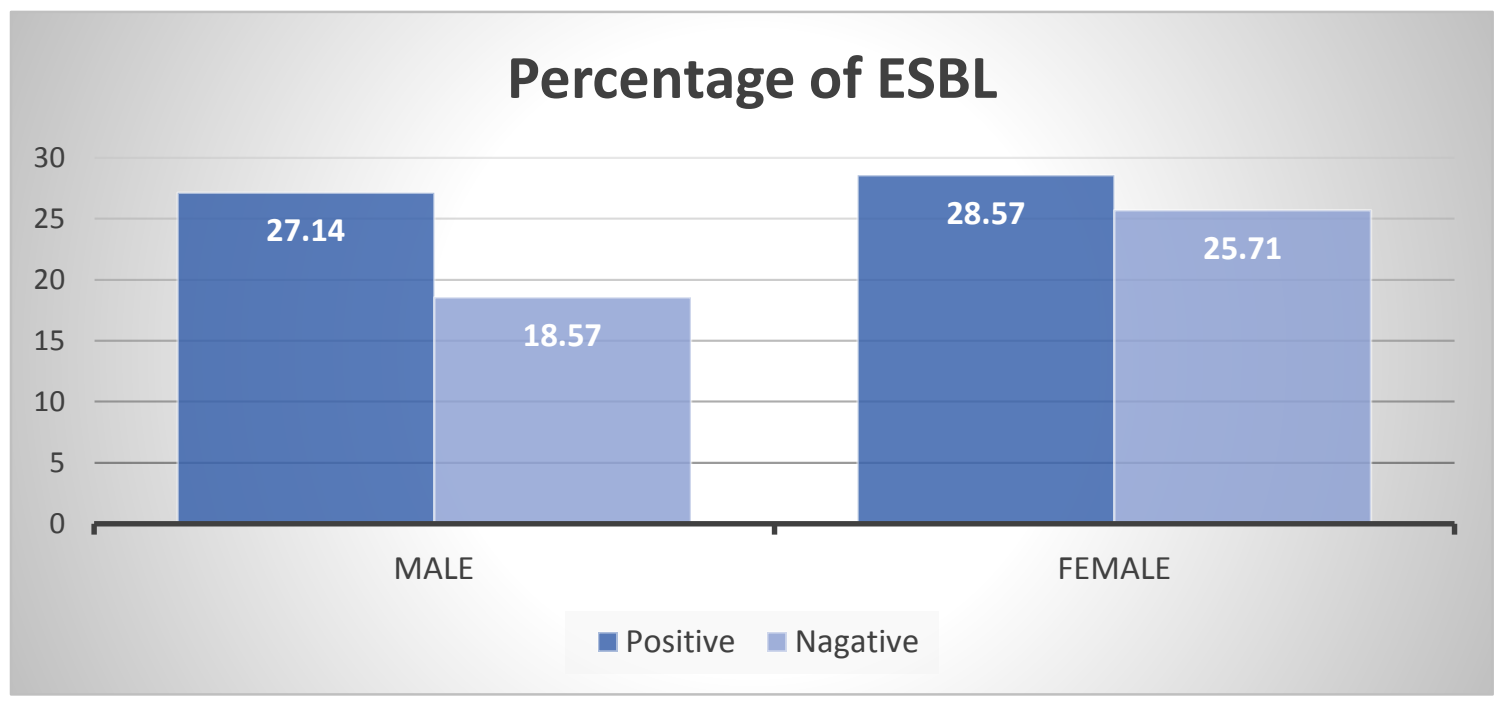

Figure 3. Shows Percentage of ESBL positive and negative in male and female 


\section{Figure 4. Shows ESBL positive strains}

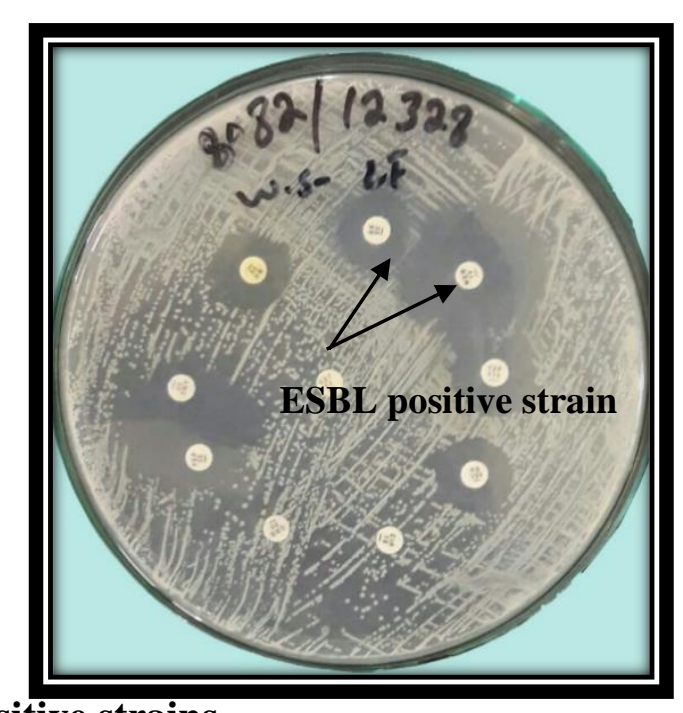

\section{Discussion}

Pakistan is a developing country with insufficient health infrastructure and poor feedback system. The solution to a problem is to understand the importance of it. In this study $E$. coli strain was isolated from various clinical samples, bearing out the suggestion that like other microorganisms. E.coli is also responsible for various clinical Infections As anticipated, the strain was resistant to Cotrimoxazole, Ciprofloxacin, Ceftriaxone, Cefepime, Amoxicillin, Tazobactam, Amikacin, Imipenem and Polymyxin b respectively, representing the appearance of multi-drug resistant forms. The present work demonstrated the resistance rate in the following direction SXT, CIP, CRO, FEP, AMC, AK or TZP, MEM and PB. Antibiogram showed a high level of resistance in the location, observed in Cotrimoxazole and Ciprofloxacin while Polymyxin b shows greater susceptibility to all the antibiotics (98.57\%). In conformity with earlier observation, our result is supported by the study of Gales et al. [18] in the USA. They observe that $99.9 \%$ of E. coli isolates were sensitive to Polymyxin $b(\mathrm{~PB})$. After Polymyxin $b$ the second most sensitive antibiotic is Imipenem (IPM) which is $90 \%$ sensitive. Our results were lower than in comparison to other study carried out in India $93.3 \%$ by Swaroop et al. [19]. It was $97 \%$ according to the study of Goudarzi et al. [20] in Iran, and $99.7 \%$ in the study conducted by Shah et al. [21] in Pakistan, moreover Ullah et al. [22] in Peshawar, Pakistan reported it is $97.4 \%$ respectively. A reason for this lower percentage of sensitivity from other studies may be due to the extensive use of antibiotics in this region. Therefore they show lower sensitivity as compared to others.

On the percentage of antibiotic resistivity, the highest resistivity is that of Co-trimoxazole (84.28\%). Our result is supported by the study of Goudarzi et al. [20] in Iran, which is $80 \%$ and Ullah et al. [22] in Peshawar, Pakistan it was $81.0 \%$.

In this study, the most important thing is ESBL which is produced by E. coli spp. This enzyme enables bacteria to cause serious infections by acquiring resistance against different kinds of antibiotics. The present study showed that out of 70 tested samples 39 $(55.71 \%)$ were ESBL positive. Our result is supported by the study carried out in Iran $(55.5 \%)$ by Goudarzi et al. [20] and in Pakistan (56.9\%) by Ullah et al. [22].

Our results were lower than in comparison to other study carried out in Turkey $84 \%$ Bali et al. [23], whereas in the study conducted by 
Ibrahim et al. [24] in Sudan reported it as $92.2 \%$, It was $67.9 \%$ in the study conducted by Fernandes et al. [25] in Portugal. Moreover, Salem et al. [26] in Egypt reported it is $87 \%$ respectively.

While our result is higher than those reported in Colombia 11.7\% Martinez et al. [27], whereas in a study conducted by Harada $e t$ al. [28] in Japan reported it as $20.4 \%$. It was $36.7 \%$ in the study conducted by $\mathrm{Yu}$ et al. [29] in China, and $13.2 \%$ in the study conducted by Kiratisin et al. [30] in Thailand. Moreover, Goudarzi et al. [20] in Saudi Arabia reported it is $30.6 \%$ respectively. The inconsistency in reported results can be attributed to the frequent use of antibiotics in that particular region which will make an organism more resistant to extendedspectrum beta-lactam antibiotics.

\section{Conclusion}

The present study shows the prevalence of ESBL in E. coli was found to be $55.71 \%$ in Lady reading hospital Peshawar. PB (Polymyxin b) (98.57\%) followed by IMP (Imipenem) (90\%) are the best choice for " $E$. coli" with the highest sensitivity. Among the resistivity of antibiotics, the highest resistivity is that of SXT (Co-trimoxazole) (84.28\%), which is the lowest acting antibiotic on these bacteria. It is concluded that the Multidrug resistance (MDR) is one of the major problems in various infections from all over the world. Several Hospitals belong to developing countries do not perform routine culture test procedure, because of limited resources, however, the present study supports the culture sensitivity test for every kind of infection, and also take some steps for reducing the resistivity, by minimizing the use of antibiotics, use of synergistic combinations, improving the hygienic measures and conduct further research to find the antibiotic pattern of resistance in E. coli.

\section{Authors' contributions}

Conceived and designed the experiments: $\mathrm{M}$ Zahid, Performed the experiments: M Afzaal, MF Afridi \& A Haseeb, Analyzed the data: M Shah, Contributed materials/ analysis/ tools: M Haroon \& R Ahmad, Wrote the paper: FU Hassan \& M Sajid.

\section{References}

1. Gonzalez CM \& Schaeffer AJ (1999). Treatment of urinary tract infection: what's old, what's new, and what works. World J Urol 17(6): 372-382.

2. Tenaillon O, Skurnik D, Picard B \& Denamur E (2010). The population genetics of commensal Escherichia coli. Nat Rev Microbiol 8(3): 207.

3. Singleton P (1999). Bacteria in biology, biotechnology, and medicine.

4. Cox-Singh J (2012). Zoonotic malaria: Plasmodium knowlesi, an emerging pathogen. Curr Opin Infect Dis 25(5): 530-536.

5. Vogt RL \& Dippold L (2005). Escherichia coli O157: H7 outbreak associated with consumption of ground beef, June-July 2002. Public Health Rep 120(2): 174-178.

6. Bentley RO \& Meganathan R (1982). Biosynthesis of vitamin $\mathrm{K}$ (menaquinone) in bacteria. Microbiol Rev 46(3): 241.

7. Russell JB \& Jarvis GN (2001). Practical mechanisms for interrupting the oralfecal lifecycle of Escherichia coli. J Mol Microb Biotech 3(2): 265-272.

8. Ogden ID, MacRae M \& Strachan NJ (2005). Concentration and prevalence of Escherichia coli $\mathrm{O} 157$ in sheep faeces at pasture in Scotland. J Appl Microbiol 98(3): 646-651.

9. Solomon EB, Pang HJ \& Matthews KR (2003). Persistence of Escherichia coli O157: H7 on lettuce plants following spray irrigation with contaminated water. J Food Protect 66(12): 21982202. 
10. Giptiyah M (2016). Efek Air Rebusan Daun Kayu Manis (Cinnamomum burmanii) Terhadap Pertumbuhan Bakteri Escherichia coli (Doctoral dissertation, University of Muhammadiyah Malang).

11. Todar R \& Pathogenic E (2007). Coli. Online Textbook on Bacteriology. University of WinkonsonMadison Department of Bacteriology.

12. Ali JA (2012). Hemolysin and Bacteriocin Production of E. coli Isolated from Urinary Tract Infection. JUBPAS 20(5): 1448-1451.

13. Bashir S, Sarwar Y, Ali A, Mohsin M, Saeed MA, Tariq A \& Haque A (2011). Multiple drug resistance patterns in various phylogenetic groups of uropathogenic E. coli isolated from Faisalabad region of Pakistan. Braz 42(4): 1278-1283.

14. Cao X, Cavaco LM, Lv Y, Li Y, Zheng B, Wang P \& Aarestrup FM (2011). Molecular Characterization and Antimicrobial Susceptibility testing of Escherichia coli isolates from urinary tract infections in 20 Chinese hospitals. J Clin Microbiol 02503.

15. Iliyasu MY, Uba A \& Agbo EB (2018). Phenotypic detection of multidrug resistant extended-spectrum betalactamase (ESBL) producing Escherichia coli from clinical samples. Afr J Cell Path 10(2): 25-32.

16. Aruna K \& Mobashshera T (2012). Prevalence of extended spectrum betalactamase production among uropathogens in South Mumbai and its antibiogram pattern. EXCLI 11: 363.

17. Begum S, Salam MA, Alam KF, Begum N, Hassan P \& Haq JA (2013). Detection of extended spectrum $\beta$-lactamase in Pseudomonas spp. isolated from two tertiary care hospitals in Bangladesh. BMC Res Notes 6(1): 7.
18. Gales AC, Jones RN \& Sader HS (2011). Contemporary activity of colistin and polymyxin $B$ against a worldwide collection of Gram-negative pathogens: results from the SENTRY Antimicrobial Surveillance Program (2006-09). J Antimicrob Chemother 66(9): 20702074.

19. Swaroop PS, Kumari PH \& Rao US (2013). Virulence-associated factors in Escherichia coli strains isolated from urinary tract infections. Int $J$ Curr Microbiol Appl Sci 2(10): 436-440.

20. Goudarzi M, Sabzehali F, Tayebi Z, Azad M, Boromandi S, Hashemi A \& Seyedjavadi SS (2014). Prevalence of blaCTX-M gene in multi-resistant Escherichia coli isolated from Urinary Tract Infections, Tehran, Iran. NBM 2(4): 107-113.

21. Shah SH (2002). Susceptibility patterns of Escherichia coli: Prevalence of multidrug-resistant isolates and extended spectrum beta-Lactamase phenotype. JPMA 52: 407.

22. Ullah F, Malik S \& Ahmed J (2009). Antibiotic susceptibility pattern and ESBL prevalence in nosocomial Escherichia coli from urinary tract infections in Pakistan. Afr J Biotechnol 8: 16.

23. Bali EB, Accedil L \& Sultan N (2010). Phenotypic and molecular characterization of SHV, TEM, CTX-M and extended-spectrum-lactamase produced by Escherichia coli, Acinobacter baumannii and Klebsiella isolates in a Turkish hospital. Afr $J$ Microbiol Res 4(8): 650-654.

24. Ibrahim ME, Bilal NE \& Hamid ME (2012). Increased multi-drug resistant Escherichia coli from hospitals in Khartoum state, Sudan. Afr Health Sci 12(3): 368-375.

25. Fernandes R, Amador P, Oliveira C \& Prudêncio C (2014). Molecular 
characterization of ESBL-producing Enterobacteriaceae in northern Portugal. Sci World J 2014.

26. Salem MM, Magdy M \& Alhosiny IM (2010). Distribution of classes 1 and 2 integrons among multi drug resistant $E$. coli isolated from hospitalized patients with urinary tract infection in Cairo, Egypt. Aust. J Basic Appl Sci 4(3): 398407.

27. Martinez P, Garzón D \& Mattar S (2012). CTX-M-producing Escherichia coli and Klebsiella pneumoniae isolated from community-acquired urinary tract infections in Valledupar, Colombia. Braz J Infect Dis 16(5): 420425.

28. Harada Y, Morinaga Y, Yamada K, Migiyama $Y$, Nagaoka $K$ \& Migiyama $Y$ (2013). Clinical and molecular epidemiology of extended-spectrum $\beta$ lactamase-producing Klebsiella pneumoniae and Escherichia Coli in a Japanese Tertiary Hospital. J Med Microb Diagn 2(127): 2161-703.

29. Yu Y, Ji S, Chen Y, Zhou W, Wei Z, Li L \& Ma Y (2007). Resistance of strains producing extended-spectrum $\beta$ lactamases and genotype distribution in China. J Infection 54(1): 53-57.

30. Kiratisin P, Apisarnthanarak A, Laesripa C \& Saifon P (2008). Molecular characterization and epidemiology of extended-spectrum- $\beta$-lactamase-

producing Escherichia coli and Klebsiella pneumoniae isolates causing health care-associated infection in Thailand, where the CTX-M family is endemic. Antimicrob Agents Chemother 52(8): 2818-2824. 